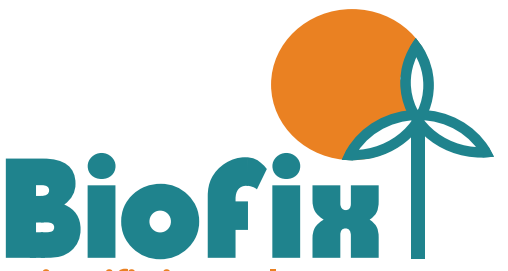

scientific journal

v. 2 n. Especial I MECFOR 2017

Recebido em 05/11/2017

Aceito em 20/11/2017

Publicado em 22/11/2017

DOI: dx.doi.org/10.5380/biofix.v2i0.56342

\title{
ASPECTOS DA QUALIDADE DO TRABALHO RELACIONADOS À EXPLORAÇÃO FLORESTAL NA AMAZÔNIA CENTRAL
}

\author{
WORK QUALITY ASPECTS RELATED TO THE \\ FOREST LOGGING IN CENTRAL AMAZON
}

\author{
Kauê Augusto Oliveira Nascimento ${ }^{1}$ \\ Fabiano Emmert ${ }^{2}$ \\ Renato César Gonçalves Robert ${ }^{3}$
}

Instituto Nacional de Pesquisas da Amazônia, Manaus, Amazonas, Brasil kaue_nascimento@hotmail.com ${ }^{1}$

Universidade Federal Rural da Amazônia, Belém, Pará, Brasil fabianoemmert@yahoo.com.br ${ }^{2}$

Universidade Federal do Paraná, Curitiba, Paraná, Brasil renatorobert@hotmail.com ${ }^{3}$

\section{RESUMO}

A baixa qualidade das operações é apontada como a principal causa da insustentabilidade ecológica do manejo florestal. Este trabalho teve como objetivo a quantificação e a análise de variáveis de qualidade no corte florestal: engate de árvores, altura de toco e árvores ocas. A área de estudo localiza-se no município de Itapiranga, Amazonas, onde a coleta de dados consistiu em 39 horas do corte florestal. Em média três árvores por jornada (12\%) engataram no momento de corte por falta de perícia. A altura média dos tocos foi de $26 \mathrm{~cm}$, com volume médio de $0,09 \mathrm{~m}^{3}$ por árvore $(2,9 \%)$ em forma de tocos. Foi encontrada, em média, $19,8 \%$ de árvores ocas por jornada, com tempo perdido de deslocamento por jornada de uma hora e 12 minutos, sendo equivalente a $12,9 \mathrm{~m}^{3}$ de produtividade perdida. As adequações do processo de planejamento da operação acarretam no aumento da segurança e do desempenho da exploração florestal.

PALAVRAS-CHAVE: Árvores ocas, Corte, Engate, Toco.

\section{ABSTRACT}

The poor quality of the operations is main causes of the ecological unsustainability of forest management. The aim of this study was the quantification and analysis of quality variables in cutting operation of logging: tree stuck, stump height and hollow trees. The study area is in the municipality of Itapiranga, Amazonas State, where the data collection consisted of 39 hours in cutting operation. On average, three trees per journey (12\%) stuck when cutting due to lack of expertise. The average height of the stumps was $26 \mathrm{~cm}$, with an average volume of $0.09 \mathrm{~m}^{3}$ per tree $(2.9 \%)$ in stumps. An average of $19.8 \%$ of hollow trees per journey, with lost displacement per journey of one hour and 12 minutes, equivalent to $12.9 \mathrm{~m}^{3}$ of lost productivity. The adequacy of the planning process of the operation increases the safety and the performance of the logging.

KEYWORDS: Hollow trees, Logging, Stuck, Stump. 


\section{INTRODUÇÃO}

Para Sabogal et al. (2006), o setor madeireiro na Amazônia contribui expressivamente para a economia regional, no entanto, enfrenta problemas com a baixa qualidade das operações florestais. Os mesmos autores constataram que há poucos profissionais qualificados, tanto para gerenciar como para trabalhar diretamente nas operações florestais. Fatores intrínsecos ao trabalho, como a alta carga física, também afetam a qualidade do trabalho florestal (AXELSSON, 1998; LILLEY et al., 2002).

Smith et al. (1985) e Silva (2007) citaram que o pagamento de bônus por produção motiva os trabalhadores a diminuir a frequência de pausas para descanso, em detrimento da qualidade e segurança do trabalho. Emmert (2014) concluiu que a baixa qualidade afeta a eficiência, causa danos desnecessários à floresta e gera perdas monetárias com desperdício de madeira. 0 relatório do projeto Biomassa e Nutrientes na Floresta Tropical (BIONTE) traz a conclusão que a baixa qualidade da exploração florestal é a principal causa da insustentabilidade ecológica do manejo florestal (PROJETO BIONTE, 1997).

Um fator importante para evitar os riscos de acidentes no trabalho florestal é o engate de árvores, sendo as árvores que após o corte permanecem em pé. Braz (2010) afirmou que a grande quantidade de cipós forma um emaranhado conectando diversas árvores e impossibilitando que árvores presas caiam. Com isso, o engate de árvores cria uma situação extremamente perigosa, o que gera a possibilidade de acidentes fatais.

O engate por imperícia do motosserrista pode estar relacionados ao cansaço e desconforto térmico, sendo citado por Ramsey (1995) e Hancock \& Vasmatzidis (2003) como um fator negativo para a perícia em atividades motoras que exigem concentração. $O$ toco é considerado a parte do fuste mais próxima ao solo, permanecendo na floresta. A quantidade varia de acordo com a espécie, podendo ou não apresentar raízes tabulares acima do solo (SILVA, 2013).

A altura de toco é um fator de qualidade do corte florestal. Entretanto, em florestas tropicais, poucos estudos definiram um padrão de qualidade na altura de corte, devido às diferentes condições de cada floresta, como composição e relevo, e aos variados diâmetros das árvores. Leite (2008) relatou que as alturas de corte em florestas na Amazônia variam entre 20 e $50 \mathrm{~cm}$.

Para Silva (2013), a estimativa do volume das árvores é a principal finalidade dos levantamentos florestais, entretanto, quando se trata dos resíduos florestais, ainda há uma carência de informações. Nas florestas tropicais há diversas espécies arbóreas que apresentam buracos ou ocos dentro do tronco, devido ao ataque de xilófagos e outros fatores. Em uma área de manejo florestal, Minette e Oliveira Filho (2000) concluíram que a presença de árvores ocas aumenta o ciclo de corte para explorar uma área e diminui a produção, devido ao baixo rendimento ou inutilização da madeira oca explorada.

Este trabalho partiu da hipótese que a melhoria do planejamento e da qualidade das operações acarretam em maior segurança e eficiência no trabalho. Sendo assim, este estudo teve o objetivo de quantificar e analisar variáveis de qualidade coletadas na operação de corte florestal.

\section{MATERIAL E MÉTODOS}

Este trabalho foi desenvolvido em uma empresa privada que executa manejo florestal seletivo na unidade de produção anual (UPA) localizada em Itapiranga, estado do Amazonas, sob as coordenadas Latitude $02^{\circ} 75^{\prime} \mathrm{S}$ e Longitude $58^{\circ} 47^{\prime} \mathrm{W}$. O corte florestal dispunha de 10 equipes com três trabalhadores cada (líder, auxiliar e motosserrista), sob carga horária de nove horas trabalhadas por jornada, os quais também recebem adicional por produção excedente.

A coleta de dados ocorreu entre os dias cinco a nove de novembro de 2015, contemplando cinco equipes diferentes em quatro jornadas completas e uma parte do dia nove de novembro. Com isso, totalizou-se 39 horas de coleta de dados.

Durante a operação, foram contabilizadas as árvores que permaneceram de pé após o corte completo do fuste (árvores engatadas). Foram coletados também altura de corte e diâmetro de toco, de forma a estimar o volume de madeira remanescente na floresta em forma de tocos.

Para os cálculos de altura de corte, foram mensuradas as alturas da parte mais baixa e mais alta do toco, sendo feita a média aritmética dessas duas medições. Os diâmetros consistiram em duas medições perpendiculares no tronco (diâmetro menor e diâmetro maior). Assim, com uso da fórmula descrita por Emmert (2014), os volumes de toco foram estimados (1).

As árvores ocas foram identificadas por meio do som gerado pela batida do facão no tronco da árvore, conhecido como teste do oco, ou pela perfuração do tronco com o sabre da motosserra. Assim, após confirmada a presença de oco, a árvore foi poupada de corte e a equipe dirigiu-se à próxima árvore marcada. Foram contabilizadas todas as árvores ocas detectadas em cada jornada de 
trabalho no corte florestal.

$$
V t=\pi \cdot \frac{\left[\frac{(d 1+d 2)}{2}\right]^{2}}{40000} \cdot\left(\frac{\frac{a 1+a 2}{2}}{100}\right)
$$

Em que:

$v t=$ volume do toco $\left(\mathrm{m}^{3}\right)$;

$d 1$ = diâmetro maior do toco $(\mathrm{cm})$;

$d 2$ = diâmetro menor do toco $(\mathrm{cm})$;

$a 1=$ altura maior do toco $(\mathrm{cm}) ; \mathrm{e}$

$a 2$ = altura menor do toco $(\mathrm{cm})$.

O tempo de deslocamento médio entre uma árvore e outra foi calculado para cada equipe analisada, sendo cronometrado por meio de um cronômetro digital Instrutemp modelo ITCD-4000. Com isso, foi possível estimar as perdas em tempo por jornada e em produtividade. As perdas em volume explorado foram calculadas com base no volume médio individual $\left(3,28 \mathrm{~m}^{3}\right)$ das árvores marcadas para corte na UPA explorada. As variáveis foram analisadas por meio de estatística descritiva, com uso do software Excel.

\section{RESULTADOS E DISCUSSÃO}

O corte foi a operação mais beneficiada por pagamento de adicional, o que interferiu diretamente no desempenho e na qualidade do trabalho, a partir do momento em que houve motivação para produzir mais. Smith et al. (1985) e Silva (2007) afirmaram que essa prática leva os trabalhadores a diminuir a frequência de pausas e a aumentar o ritmo de trabalho, com consequências negativas sobre qualidade do trabalho.

Com isso, deve-se estabelecer novos modelos para o pagamento adicional por produção, de forma a equilibrar a produtividade com aspectos importantes, como qualidade, saúde e segurança do trabalho, assim como enfatizados por Axelsson (1998) e Lilley et al. (2002). Uma das alternativas para isso é o estabelecimento de uma produtividade limite para pagamento de adicional.

Não foram observados fatores ambientais (cipós) como causadores do engate das árvores. Contudo, verificou-se que para as três jornadas de trabalho analisadas quanto ao engate de árvores, em média três árvores por jornada (12\%) engataram nas copas por imperícia ou inexperiência dos motosserristas. Entretanto, Batista (2008) constatou experiência e bom treinamento dos trabalhadores, corroborado pelos resultados de Emmert (2014).

Porém, observou-se neste estudo que os trabalhadores recém contratados foram alocados para o trabalho com motosserra, sendo treinados pelos trabalhadores experientes durante a própria atividade, o que demonstrou mudanças organizacionais na empresa. Essas práticas ajudaram a explicar o alto índice de árvores engatadas, falhas na qualidade do trabalho, geração de risco grave de acidentes e perdas de tempo nas operações de corte e pré-arraste.

Encontrou-se média de $0,09 \mathrm{~m}^{3}$ por toco no presente estudo, sendo aproximadamente metade dos 0,16 $\mathrm{m}^{3}$ observados por Emmert (2014) e próximo de 0,12 $\mathrm{m}^{3}$ mensurado por Silva (2013), cujos estudos foram realizados na mesma área de manejo florestal, porém em UPAs diferentes. Silva (2013) também estimou os volumes dos tocos, resultando em média de $2 \%$. Além disso, encontrou-se neste estudo resíduo médio de $2,9 \%$ toco por árvore e estimado volume total de madeira deixado em campo equivalente em árvores, com base na média de volume individual (Tabela 1).

Tabela 1. Volume de madeira deixado em campo em forma de tocos por jornada de trabalho

\begin{tabular}{cccccc}
\hline Jornada & $\boldsymbol{d}(\mathbf{c m})$ & $\boldsymbol{h}(\mathbf{c m})$ & $\boldsymbol{v}\left(\mathbf{m}^{\mathbf{3}}\right)$ & $\begin{array}{c}\text { Proporção } \\
\text { resíduos }\end{array}$ & $\begin{array}{c}\text { Árvores } \\
\text { equivalente }\end{array}$ \\
\hline 1 & 67,77 & 24,55 & 3,37 & $2,94 \%$ & 1,0 \\
2 & 63,10 & 25,69 & 1,84 & $2,57 \%$ & 0,6 \\
3 & 65,11 & 25,05 & 1,47 & $3,06 \%$ & 0,4 \\
4 & 65,96 & 28,75 & 2,59 & $2,94 \%$ & 0,8 \\
\hline Média & $\mathbf{6 5 , 4 8}$ & $\mathbf{2 6 , 0 1}$ & $\mathbf{2 , 3 2}$ & $\mathbf{2 , 8 7 \%}$ & - \\
\hline
\end{tabular}

$d=$ diâmetro médio; $h=$ altura média; e $v=$ volume total.

Na primeira jornada de trabalho analisada, o volume residual foi significativamente maior, possivelmente devido ao maior número de árvores derrubadas por essa equipe (produtividade) ou em decorrência do maior diâmetro das árvores. De forma geral, foram deixados 2,3 $\mathrm{m}^{3}$ de madeira por jornada de trabalho na forma de tocos. Para todos os tocos mensurados, estimou-se altura média de $26 \mathrm{~cm}$, sendo relativamente menor que a média $29,6 \mathrm{~cm}$ de observada por Emmert (2014).

Os valores de altura de toco podem ser influenciados pela postura inadequada e pelas dores musculoesqueléticas oriundas da repetitividade dessa operação ao longo da jornada, como enfatizado por Sanches (2012). Contudo, a empresa não define padrões de qualidade para a altura de corte, em que há muitos motosserristas inexperientes, o que demonstra a oportunidade de melhorias no processo de qualidade do corte.

Foram contabilizadas as árvores ocas encontradas nas jornadas de trabalho, bem como o tempo de deslocamento 
perdido até elas e a porcentagem de árvores ocas em relação ao número de árvores derrubadas, como demonstrados na Tabela 2.

Em média, 12,9 $\mathrm{m}^{3}$ foram perdidos por jornada de trabalho em função do tempo gasto em deslocamentos até árvores ocas. Com isso, poderiam ser derrubadas aproximadamente quatro árvores a mais por jornada de trabalho, considerando a identificação prévia das árvores ocas durante o inventário. Em média, 19,8\% das árvores marcadas para corte continham oco. Esses resultados condizem com os encontrados por Minette \& Oliveira Filho (2000), Batista (2008) e Emmert (2014).

$\mathrm{O}$ deslocamento entre as árvores foi uma das atividades parciais com maior demanda de tempo, afetando diretamente o rendimento do trabalho, conforme Batista
(2008) e Emmert (2014). O deslocamento até árvores ocas durou cerca de uma hora e 12 minutos por jornada, equivalendo a tempo e energia desperdiçados que certamente afetaram a produtividade. Esses resultados são corroborados por Batista (2008) e Sanches (2012), ao concluirem que essas circunstâncias aceleram o processo de fadiga e o desconforto térmico, com consequente queda de rendimento, agravado pelo peso da motosserra carregada.

Dessa forma, pode-se afirmar que são necessárias melhorias no planejamento e na qualidade das operações de manejo florestal, com o objetivo de aumentar o desempenho e a produtividade do trabalho, especialmente na operação de corte, conforme corroborado por Emmert (2014).

Tabela 2. Produtividade perdida pelo corte florestal no deslocamento até árvores ocas

\begin{tabular}{|c|c|c|c|c|c|c|}
\hline Equipe & $\begin{array}{c}\text { Número } \\
\text { árvores ocas }\end{array}$ & $\begin{array}{l}\text { Produtividade } \\
\text { média }\left(\mathrm{m}^{3} / \mathrm{h}\right)\end{array}$ & $\begin{array}{c}\text { Tempo em } \\
\text { deslocamento/árvore }\end{array}$ & $\begin{array}{c}\text { Tempo perdido em } \\
\text { deslocamento }\end{array}$ & $\begin{array}{c}\text { Produtividade } \\
\text { perdida }\end{array}$ & \% Árvores ocas \\
\hline 1 & 6 & 13,97 & $00: 12: 43$ & 01:16:19 & 17,69 & 20,69 \\
\hline 2 & 2 & 9,33 & $00: 18: 38$ & 00:37:15 & 5,75 & 8,70 \\
\hline 3 & 5 & 6,36 & $00: 21: 36$ & 01:47:58 & 11,45 & 27,78 \\
\hline 4 & 6 & 11,43 & $00: 14: 45$ & $01: 28: 27$ & 16,76 & 22,22 \\
\hline $5^{*}$ & 2 & 11,05 & $00: 08: 22$ & - & - & - \\
\hline Média & 4,75 & 10,43 & $00: 15: 13$ & 01:12:15 & 12,91 & 19,84 \\
\hline
\end{tabular}

* Os números absolutos da equipe 5 não foram considerados, pois o turno de trabalho dessa equipe findou às 10 horas.

\section{CONCLUSÕES}

As adequações do planejamento e da organização da operação de corte acarretam no aumento da segurança, da qualidade e do desempenho da exploração florestal na Amazônia central.

\section{AGRADECIMENTOS}

Ao Laboratório de Manejo Florestal (LMF-INPA), pela contribuição na elaboração da pesquisa.

À Mil Madeiras Preciosas Ltda., pela colaboração logística e suporte na coleta de dados.

Ao Instituto Nacional de Pesquisas da Amazônia (INPA) e ao Conselho Nacional de Desenvolvimento Científico e Tecnológico (CNPq), pela concessão da bolsa de estudos.

\section{REFERÊNCIAS}

AXELSSON, S. A. The mechanization of logging operations in Sweden and its effect on occupational safety and health. International Journal of Forest Engineering, v. 9, p. 25-31, 1998.

BATISTA, H. L. D. P. Estudo de tempo e rendimento da motosserra considerando fatores ergonômicos numa exploração florestal na Amazônia Central. 2008. 105 f. Dissertação (Mestrado em Ciências de Florestas Tropicais) Instituto Nacional de Pesquisas da Amazônia, Universidade Federal do Amazonas, Manaus, 2008.

BRAZ, E. M. Subsídios para o planejamento do manejo de floresta tropicais da Amazônia. 2010. 237 f. Tese (Doutorado em Ciências Florestais) - Universidade Federal de Santa Maria, Santa Maria, 2010.

EMMERT, F. Combinação de dados de campo e métodos computacionais para o planejamento da exploração florestal na Amazônia. 2014. 190 f. Tese (Doutorado em Ciências Florestais) - Universidade de Brasília, Brasília, 2014.

HANCOCK, P. A.; VASMATZIDIS, I. Effects of heat stress on cognitive performance: the current state of knowledge. International Journal Hyperthermia, v. 19, n. 3, p. 355-372. 2003.

LEITE, F. S. Estimativa do volume de madeira a partir do diâmetro da cepa em uma área explorada de floresta amazônica de terra firme. 2008. 74 f. Dissertação (Mestrado em Ciências Florestais) - Universidade de Brasília, Brasília, 2008.

LILLEY, R.; FEYER, A. M.; KIRK, P.; GANDER, P. A. Survey of forest workers in New Zealand - Do hours of work, rest, and recovery play a role in accidents and injury? Journal of Safety Research, v. 33, p. 53-71, 2002. 
MINETTE, L. J.; OLIVEIRA FILHO, R. F. Análise técnica e econômica do corte florestal planejado de floresta tropical úmida de terra firme na Amazônia Ocidental. Revista Arvore, v. 24, n. 4, p. 429435, 2000.

PROJETO BIONTE. Relatório final projeto BIONTE - Biomassa e nutrientes na floresta tropical. Manaus: INPA/DFID, 1997. 345 p.

RAMSEY, J.D. Task performance in heat: A review. Ergonomics, v. 38, n. 1, p. 154-165, 1995.

SABOGAL, C.; LENTINI, M.; POKORNY, B.; SILVA, J. N. M.; ZWEEDE, J.; VERÍSSIMO, A.; BOSCOLO, M. Manejo florestal empresarial na Amazônia brasileira. Belém: CIFOR, 2006. 74 p.

SANCHES, A. L. P. Metas de produção em função da carga física do trabalho e repetitividade para operações de colheita florestal em terrenos montanhosos. 2012. 127 f. Dissertação (Mestrado em Ciência Florestal) - Universidade Federal de Viçosa, Viçosa, 2012.

SILVA, E. P. Avaliação dos fatores ergonômicos em operações de extração florestal em terrenos montanhosos na região de Guanhães, MG. 2007. 125 f. Dissertação (Mestrado em Ciência Florestal) - Universidade Federal de Viçosa, Viçosa, 2007.

SILVA, A. R. Volumetria de resíduos da operação de corte em floresta manejada. 2013. 50 f. Dissertação (Mestrado em Ciências Florestais e Ambientais) - Universidade Federal do Amazonas, Manaus, 2013.

SMITH, L. A.; WILSON, G. D.; SIROIS, D. L. Heart-rate response to forest harvesting work in the South-Eastern United States during summer. Ergonomics, v. 28, n. 4, p. 655-664, 1985. 